\title{
A Case report of coronavirus disease-19-associated pulmonary aspergillosis and pneumothorax
}

\author{
Maruteesh Mallappa ${ }^{1}$, Pradeep Rangappa ${ }^{2}$, Ipe Jacob ${ }^{3}$, Rajesh V Helavar ${ }^{4}$, Karthik Rao \\ From ${ }^{1}$ Registrar, ${ }^{2}$ Consultant, ${ }^{3}$ Registrar, Department of Critical Care, Consultant, ${ }^{4}$ Department of Radiology, ${ }^{5}$ Department of Critical Care, Columbia \\ Asia Referral Hospital, Bengaluru, Karnataka, India
}

\begin{abstract}
Secondary infections in coronavirus disease (COVID)-19 pneumonia in association with lung cavitation are rare findings. The development of cavitation has been attributed to aseptic liquefaction of pulmonary thromboemboli or secondary infection, resulting in the formation of pneumatoceles. Rupture of a large pneumatocele may lead to the development of pneumothorax. We report the case of a 56-year-old male who was treated for COVID-19 pneumonia with severe acute respiratory distress syndrome (ARDS) but developed pneumothorax and a cavitary lesion in the recovery period. This was further complicated by a co-infection with Aspergillus fumigatus in endotracheal secretions. The pneumothorax failed to resolve and there was a failure to wean off mechanical ventilation, culminating in death after 2 weeks. This case report highlights the need to monitor for small pneumatoceles even in the recovery phase of COVID-19 which may predispose to pneumothorax or pneumomediastinum.
\end{abstract}

Key words: Aspergillus fumigatus, Coronavirus disease-19, Pneumonia, Secondary infections

$\mathrm{S}$ econdary infections are not uncommon in coronavirus disease (COVID)-19 patients and their incidence range from $9 \%$ to as high as $58 \%$ [1,2]. Early admission to intensive care unit (ICU), invasive mechanical ventilation, severe hypoxemia (low $\mathrm{PaO}_{2} / \mathrm{FiO}_{2}$ ratio), and severe lymphopenia have been identified as risk factors for secondary infections. The most common infections seen are respiratory, blood-stream, and urinary infections. In respiratory infections, the most detected pathogens were Gram-negative bacteria (50\%), followed by Gram-positive bacteria (27\%), viruses (12\%), fungi (8\%), and others (4\%) [2]. A noteworthy proportion of patients has been found to have multiple isolates, as in the present case, which grew Aspergillus fumigatus in endotracheal secretions, Stenotrophomonas maltophilia in pleural fluid, and candida in urine [2].

\section{CASE REPORT}

A 56-year-old male, known diabetic and hypertensive, was referred to our tertiary hospital ICU with a complaint of severe dyspnea for 1 week. He was diagnosed with COVID-19 pneumonia with severe ARDS 1 month back at a rural hospital and treated in ICU with remdesivir, enoxaparin, dexamethasone, and oseltamivir,

\section{Access this article online}

Received - 19 February 2021

Initial Review - 15 March 2021

Accepted - 05 April 2021

DOI: $10.32677 / I J C R .2021 . v 07 . i 04.007$ along with non-invasive positive pressure ventilation (NIPPV). He had a prolonged ICU course of about 4 weeks, after which a repeat COVID-19 real-time fluorescent polymerase chain reaction (RT-PCR) test was negative and then, he was shifted to the ward. About 2 days later, he had a sudden onset of chest pain and breathlessness. Chest X-ray showed a left-sided pneumothorax. An underwater seal drain (UWSD) was inserted into the left lung and he was shifted to our hospital for further management.

At presentation, he was conscious and oriented, afebrile, with a heart rate of $100 \mathrm{bpm}$, blood pressure of 118/80 mm Hg, respiratory rate of $26 / \mathrm{min}$, and oxygen saturation of $94 \%$ with $15 \mathrm{~L} /$ min oxygen through a non-rebreather mask; hence, he was initiated on NIPPV. He had an Acute Physiology and Chronic Health Evaluation II score of 17 with a predicted mortality rate of $26 \%$.

COVID-19 RT-PCR was repeated, which was negative. Computed tomography (CT) pulmonary angiogram ruled out pulmonary thromboembolism but showed a persisting left pneumothorax with the chest tube in situ, extensive pulmonary fibrosis, and large, thick-walled cavitation in the left lower lobe (Figs. 1a-c); hence, a second left-sided UWSD was inserted. His total leukocyte count at admission was $32,900 \mathrm{cells} / \mathrm{mm}^{3}$.

The patient was started on piperacillin-tazobactam $4.5 \mathrm{~g} 4$ times daily pending cultures. Other medications included pentoxifylline

Correspondence to: Dr. Pradeep Rangappa, Department of Critical Care, Columbia Asia Referral Hospital, Yeshwantpur, Bengaluru - 560 055, Karnataka, India. E-mail: prangap939@gmail.com

(C) 2021 Creative Commons Attribution-NonCommercial 4.0 International License (CC BY-NC-ND 4.0). 
$400 \mathrm{mg}$ twice daily and pirfenidone $400 \mathrm{mg}$ thrice daily. In view of worsening dyspnoea, he was intubated and mechanically ventilated on day 2 in ICU. Sputum smear was negative for acidfast bacilli and there were no features suggestive of vasculitis. Deep endotracheal swab cultures grew A. fumigatus and he was started on voriconazole $200 \mathrm{mg}$ once daily intravenously. He was tracheostomised on day 7 in ICU as he failed all attempts to wean off mechanical ventilation. Although serial chest X-rays showed expansion of the left lung, there was a persistent air leak through both intercostal drains. CT scan of the chest was repeated which showed an increase in the pneumothorax and no change in the cavitation (Figs. 2a-c). There was also no sign of bronchopleural fistula. Negative pressure was applied to the chest drains. Cardiothoracic opinion was to continue conservative management in view of his unstable status.

The patient continued to deteriorate clinically with a gasping pattern of breathing, desaturating to unsafe levels, needing high $\mathrm{FiO}_{2}$ and positive end-expiratory pressure, and vasopressor support. He became increasingly drowsy, with type II respiratory failure and worsening renal function, requiring renal replacement therapy. He eventually succumbed to the illness on day 14 of the ICU stay.

\section{DISCUSSION}

CT scan findings suggestive of possible COVID-19-associated pulmonary Aspergillosis (CAPA) such as the halo sign, aircrescent sign, or cavitation (as seen in the present case) are rare and should provoke testing for Aspergillus [3]. The mechanisms by which viruses render host defenses susceptible to secondary infections include impairment of mucociliary clearance leading to bacterial colonization and generation of pro-inflammatory molecules such as tumor necrosis factor-alpha. In particular, postviral desensitization of lung sentinel cells to toll-like receptor signals might contribute to secondary bacterial pneumonia associated with viral infection [4].

The patient in this case report met the modified Asp ICU criteria for Invasive Pulmonary Aspergillosis [5], including dyspnea, worsening respiratory insufficiency, pulmonary infiltrates on imaging, and positive culture for A. fumigatus. This is an opportunistic pathogen and typically infects immunosuppressed patients such as those with severe prolonged neutropenia, inherited or acquired immunodeficiency, steroid dependency, chemotherapy medications, and transplant patients. The use of immunomodulatory drugs such as anakinra (recombinant interleukin-1Ra), tocilizumab (anti-interleukin-6), and Janus kinase inhibitors may also predispose patients to CAPA [3].

In view of the high morbidity and mortality, early diagnosis and treatment are imperative. Ideally, screening for CAPA entails using a combination of $\mathrm{CT}$ chest imaging and Aspergillus antigen tests on bronchoalveolar lavage (BAL) and serum, including galactomannan enzyme-linked immunosorbent assay or lateralflow tests, or Aspergillus PCR [3]. As BAL is an aerosolgenerating procedure, an alternative is the $\beta$-D-glucan.

Therapeutic strategies consist of prophylaxis in risk groups and the early application of antifungal agents in suspected or probable disease [6]. Amphotericin B desoxycholate (1-1.5 mg/kg/day) or lipid formulation $(1-3 \mathrm{mg} / \mathrm{kg} /$ day $)$ is the current standard medication in invasive aspergillosis. Percutaneous CT-guided local application of $\mathrm{AmB}$ has been proven to be effective.
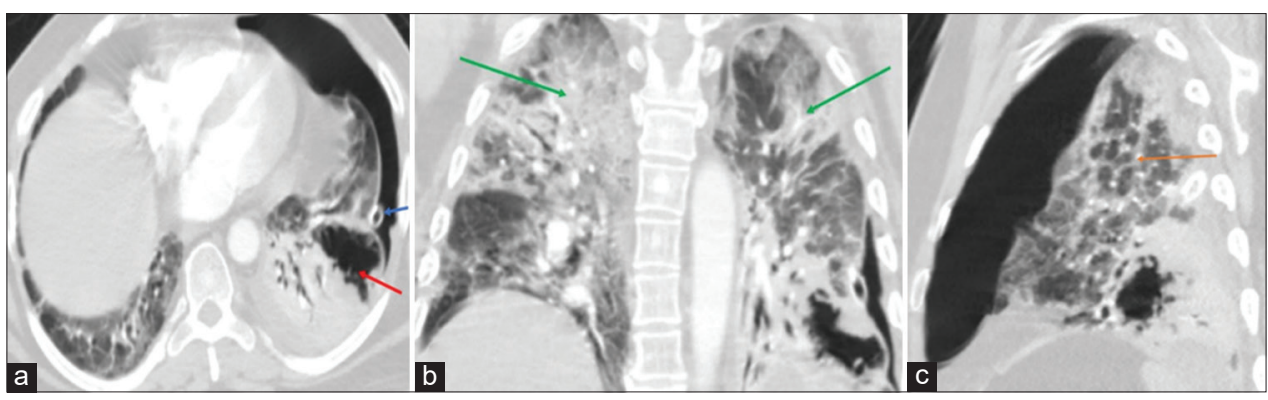

Figure 1: Computed tomography pulmonary angiogram chest. (a) Axial view: Blue arrow shows large left pneumothorax with intercostal drain in situ. Red arrow shows cavity in the left lower lobe about $5 \mathrm{~cm} \times 4 \mathrm{~cm}$ in size; (b) coronal view: Green arrows show extensive bilateral fibrosis and consolidation and cavitation in base of the left lung; (c) Sagittal view of the left lung showing large pneumothorax. Orange arrow shows interlobular thickening
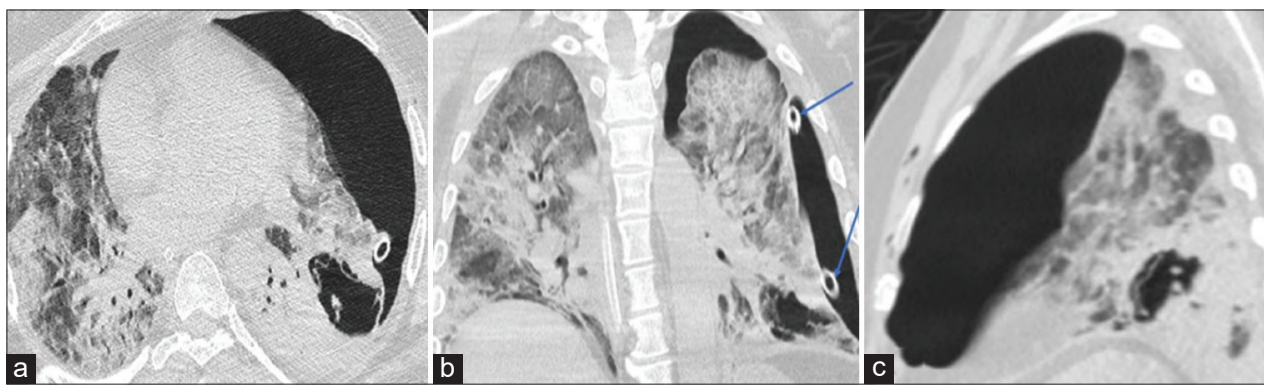

Figure 2: Repeat computed tomography chest after 4 days. (a) Axial view showing persisting large left pneumothorax; (b) coronal view with blue arrows showing two intercostal drains in situ; (c) sagittal view of the left lung 
Alternatives are the new azole derivatives, such as itraconazole, voriconazole, and the new echinocandins. Voriconazole is effective against all Aspergillus species and given at a dose of $200 \mathrm{mg}$ twice daily orally. Typical side effects include visual disturbances, hepatotoxicity, and dermal rash.

In the present case, virus-induced inflammation is causing obstruction and cystic dilation of the bronchi as well as prolonged mechanical ventilation with high tidal volumes and subsequent rupture of alveolar and intra-acinar septa may have led to the formation of pneumatocele. Biopsy of the COVID-19 lung has shown pus collection within the cystic dilations of the lung parenchyma, remnants of bronchiolar epithelium, and squamous metaplasia. Rupture of a subpleural pneumatocele, in turn, may lead to pneumothorax $[7,8]$.

Pneumatoceles are thin-walled, air-filled parenchymal cysts within the lung that can complicate severe pneumonia. They can develop over a short period of 12 days from the liquefaction and drainage of necrotic lung tissue, allowing passage of air into the interstitial space [9]. Severe Acute Respiratory Syndrome - coronavirus-2-related pneumatoceles may be multiple and resemble emphysema [8]. Their size is also highly variable, with some forming giant bullae. Either in the context of multiple pneumatoceles or in the context of the isolated giant bulla, pneumothorax is a predictable complication. Lung cavitation can also occur due to aseptic liquefaction or secondary infection of pulmonary infarcts [10]. Pulmonary infarction may occur secondary to pulmonary emboli, which may complicate up to $30 \%$ of cases of COVID-19 pneumonia [11]; however, in the present case, there was no evidence of pulmonary embolism.

Certain disease processes increase the risk of barotrauma significantly, including ARDS as seen in our patient, chronic obstructive pulmonary disease, asthma, interstitial lung disease, and Pneumocystis jirovecii pneumonia. Infection with cavityforming organisms such as Mycobacterium tuberculosis, Klebsiella pneumoniae, and Streptococcus pneumoniae should be ruled out as well as any vasculitic process causing cavitation.

Cases of COVID 19 with aspergillosis (CAPA), complicated by pneumothorax, have been rarely reported in the literature. In a study of five patients with CAPA [12], the most common sign on CT scan of the chest was nodular infiltrates; cavities were seen in only two cases; and mortality was $60 \%$. In the present case, the ventilator settings were aimed to limit peak pressures and keep plateau pressures below $30 \mathrm{~cm}$ water. In this setting, the cause of death could be attributed to the Aspergillus infection, acidosis, and non-pulmonary organ failure with a contribution by the non-resolving pneumothorax, as corroborated by other studies [13]. Persistent pneumothorax and air leak should prompt surgical referral, according to 2010 guidelines from the British Thoracic Society [14]. In a large case series of COVID-19 with pneumothorax [15], one patient who had a persistent air leak was despite two chest drain insertions, similar to our case, was successfully treated with bullectomy and pleurodesis.

\section{CONCLUSION}

CT scan of the chest in patients with severe COVID-19 during the recovery period is important to rule out small lung cavities, which, if ignored, may prove detrimental to the patient. With the ubiquitous use of dexamethasone in the treatment of COVID-19 pneumonia, it is prudent to remain vigilant for signs of polymicrobial infections, including opportunistic organisms such as aspergillosis, and initiate early treatment.

\section{REFERENCES}

1. Langford BJ, So M, Raybardhan S, Leung V, Westwood D, MacFadden DR, et al. Bacterial co-infection and secondary infection in patients with COVID-19: A living rapid review and meta-analysis. Clin Microbiol Infect 2020;26:1622-9.

2. Zhang H, Zhang Y, Wu J, Li Y, Zhou X, Li X, et al. Risks and features of secondary infections in severe and critical ill COVID-19 patients. Emerg Microbes Infect 2020;9:1958-64.

3. Armstrong-James D, Youngs J, Bicanic T, Abdolrasouli A, Denning DW, Johnson E, et al. Confronting and mitigating the risk of COVID-19 associated pulmonary aspergillosis. Eur Respir J 2020;56:2002554.

4. Hendaus MA, Jomha FA. COVID-19 induced superimposed bacterial infection. J Biomol Struct Dyn 2020;2020:1-7.

5. Schauwvlieghe AF, Rijnders BJ, Philips N. Invasive aspergillosis in patients admitted to the intensive care unit with severe influenza: A retrospective cohort study. Lancet Respir Med 2018;6:782-92.

6. Reichenberger F, Habicht JM, Gratwohl A, Tamm M. Diagnosis and treatment of invasive pulmonary aspergillosis in neutropenic patients. Eur Respir J 2002;19:743-75.

7. Castiglioni M, Pelosi G, Meroni A, Tagliabue M, Uslenghi E, Salaris D, et al. Surgical resections of superinfected pneumatoceles in a COVID-19 patient. Ann Thorac Surg 2021;111:e23-5.

8. Jolobe OM. Air leaks, pneumatoceles, and air spaces in COVID-19 pneumonia. Am J Emerg Med 2020;2020:30792.

9. Quigley MJ, Fraser RS. Pulmonary pneumatocele: Pathology and pathogenesis. AJR Am J Roentgenol 1988;150:1275-7.

10. Libby LS, King TE, LaForce FM, Schwarz MI. Pulmonary cavitation following pulmonary infarction. Medicine (Baltimore) 1985;64:342-8.

11. Leonard-Lorant I, Delabranche X, Severac F, Helms J, Pauzet C, Collange $\mathrm{O}$, et al. Acute pulmonary embolism in patients with COVID-19 at CT angiography and relationship to d-dimer levels. Radiology 2020;296:E189-91.

12. Koehler P, Cornely OA, Böttiger BW, Dusse F, Eichenauer DA, Fuchs F, et al. COVID-19 associated pulmonary aspergillosis. Mycoses 2020;63:528-34.

13. Schnapp LM, Chin DP, Szaflarski N, Matthay MA. Frequency and importance of barotrauma in 100 patients with acute lung injury. Crit Care Med 1995;23:272-8.

14. MacDuffA, Arnold A, Harvey J. Management of spontaneous pneumothorax: British thoracic society pleural disease guideline 2010. Thorax 2010;65 Suppl 2:ii18-31.

15. Martinelli AW, Ingle T, Newman J, Nadeem I, Jackson K, Lane ND. COVID-19 and pneumothorax: A multicentre retrospective case series. Eur Respir J 2020;56:2002697.

\section{Funding: None; Conflicts of Interest: None Stated.}

How to cite this article: Mallappa M, Rangappa P, Jacob I, Helavar RV, Rao K. A Case Report of Coronavirus disease-19associated Pulmonary Aspergillosis and Pneumothorax. Indian J Case Reports. 2021;7(4):146-148. 